\title{
Molecular insight: SERPINB3 and AR sensitivity might reduce the risk of COVID-19 infection and complications in prostate cancer patients receiving androgen-deprivation therapy
}

\author{
Shiv Verma ${ }^{1,2}$, Sanjay Gupta ${ }^{1,2,3,4}$ \\ 'Department of Urology, Case Western Reserve University, School of Medicine, Cleveland, OH 44106, USA. \\ ${ }^{2}$ The Urology Institute, University Hospitals Cleveland Medical Center, Cleveland, OH 44106, USA. \\ ${ }^{3}$ Department of Nutrition, Case Western Reserve University, Cleveland, OH 44106, USA. \\ ${ }^{4}$ Division of General Medical Sciences, Case Comprehensive Cancer Center, Cleveland, OH 44106, USA.
}

Correspondence to: Dr. Sanjay Gupta, Department of Urology, Case Western Reserve University, School of Medicine, 10900 Euclid Avenue, Cleveland, OH 44106, USA. E-mail: Sanjay.gupta@case.edu

\begin{abstract}
How to cite this article: Verma S, Gupta S. Molecular insight: SERPINB3 and AR sensitivity might reduce the risk of COVID-19 infection and complications in prostate cancer patients receiving androgen-deprivation therapy. J Trans/ Genet Genom 2021;5:288-91. https://dx.doi.org/10.20517/jtgg.2021.30
\end{abstract}

Received: 7 Jun 2021 Accepted: 9 Jun 2021 First online: 17 Jun 2021

Academic Editor: Sanjay Gupta Copy Editor: Xi-Jun Chen Production Editor: Xi-Jun Chen

\section{INTRODUCTION}

SARS-CoV-2 (COVID-19) entry into host cells is facilitated by the transmembrane protease TMPRSS2 $2^{[1]}$, which is expressed in both the lungs and prostate tissue ${ }^{[2]}$. Pre-clinical data suggest that TMPRSS2 expression can be modulated by the androgen receptor $(\mathrm{AR})^{[3]}$ and that androgen deprivation therapy (ADT) might protect patients from SARS-CoV-2 infection and reduces disease severity ${ }^{[4]}$. In a longitudinal study, Montopoli et al ${ }^{[5]}$, demonstrated that prostate cancer patients receiving ADT were likely to have four times less SARS-CoV-2 infection rates and disease severity compared with non-ADT patients. This study was supported by Patel et al. ${ }^{[4]}$, where 58 prostate cancer patients infected with SARS-CoV-2 exhibited low disease severity and concluded that ADT might limit the severity of COVID-19 infection. However a study from Kwon et al. ${ }^{[6]}$, was in disagreement with the above findings and revealed no association between ADT treatment and its protection against COVID-19 infection. Subsequently, Klein et al.$^{[7]}$ studying a cohort of prostate cancer patients who received ADT, the percentage rates of positive and negative COVID-19 cases 
were almost the same, 5.6\% and 5.8\% $(\mathrm{OR}=0.93 ; P=0.8)$, highlighting that $\mathrm{ADT}$ treatment does not appear to be protective against COVID-19 infection.

\section{MOLECULAR INSIGHT}

Studies referenced above are solely based on clinical observations on prostate cancer patients with or without COVID-19 infection and their association with ADT, however, the molecular insight(s) are unknown. To address the above, we performed a meta-analysis utilizing a database comprising of human prostate cancer patients who received ADT (GSE150368) ${ }^{[8]}$ and compared with patients who did not receive ADT (GSE69223) ${ }^{[9]}$. Our rationale was based on AR sensitivity and the non-genomic function of AR. It is known that AR regulates the transcription of the transmembrane protease, serine 2 (TMPRSS2) required for SARS-CoV-2 infectivity ${ }^{[10]}$. Overlaying of the prostate cancer ADT database with COVID-19 disease showed significant interaction ( $P=1.29 \mathrm{E}-26)$, while patients with no ADT did not exhibit any interaction. Interestingly, the analysis identified genes associated with COVID-19 viz. Serpin Peptidase Inhibitor, Clade B (Ovalbumin), and Member 3 (SERPINB3) showing increase expression (5.8-fold; $P=7.50 \mathrm{E}-11$ ) after ADT treatment and a high degree of interaction with COVID-19 [Figure 1].

In humans, SERPINB3 is expressed in a high level in the nasopharynx, bronchus membrane along with other cellular subsets and across tissues ${ }^{[11]}$. Functionally, SERPINB3 activates NF- $\kappa B$ and the expression of other pro-inflammatory cytokines ${ }^{[12]}$, predominantly IL- $6^{[13]}$, which leads to an epithelial-mesenchymal transition (EMT)-like phenotypic changes that might respond as the first line of defense against COVID-19 infection. The SERPIN gene encodes plasminogen activator inhibitor (PAI). Reports suggest that PAI-2 expression markedly reduces the surface expression of the virus receptor molecules viz. CD55 (DAF), constitutive androstane receptor, and intercellular adhesion molecule 1 and thus inhibits the binding of the virus to the cellular membrane. Lastly, we predict that SERPINB3 has the ability to bind with TMPRSS2 at serine and threonine sites that may intervene or block the COVID-19 entry into the cell and inhibits its infectivity.

\section{CONCLUSION}

Elevated expression of SERPINB3 favors a role for initiation of the acute inflammatory response. SERPINB3 might serve as promising prophylactic to inhibit the progression and severity of COVID-19, by hindering the entry of SARS-CoV-2, in part, via TMPRSS2 and inhibiting consequent inflammation, coagulopathies, and multiple organ failure.

\section{STATISTICAL ANALYSIS}

The GSE database was analyzed using GEO2R and IPA, and the absolute value of log2 fold change greater than one was used, and an adjusted $P$-value less than 0.05 was considered as differentially expressed. Differentially expressed genes (DEGs) were overlaid with the global molecular network in the Ingenuity Pathway Knowledge Base (IPKB). IPA was performed to identify diseases and functions, and gene networks that are significant to RNA-Sequencing outcomes and to categorize DEGs in specific diseases and functions such as COVID-19. 


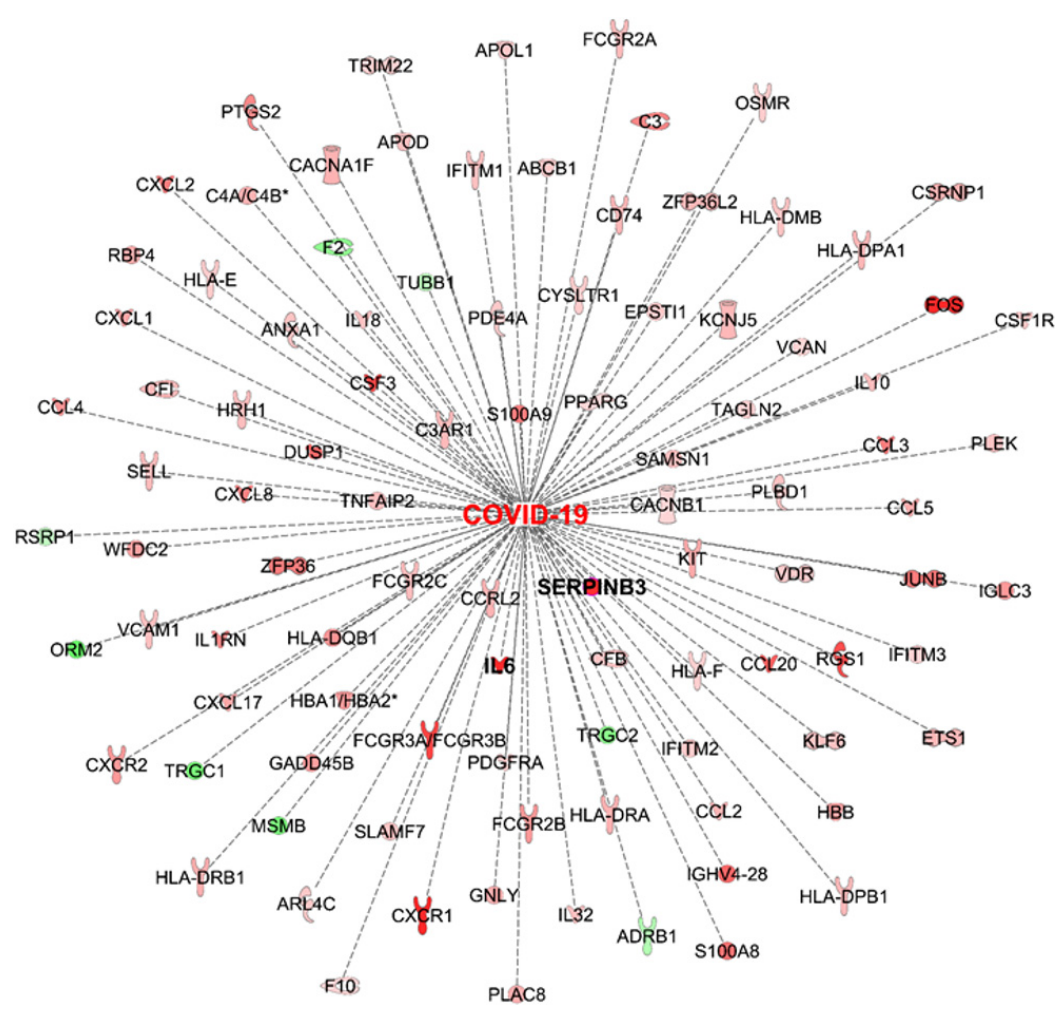

Figure 1. Differentially expressed genes of RNA-Seq. data of prostate cancer patients who received ADT was overlaid with COVID19, demonstrating significant interaction $(P=1.29 \mathrm{E}-26)$ between them with 98 molecules overlapped. Among them the expression of SERPINB3, IL6, FOS, FCGR3A/B, CXCR1 were significantly upregulated and highlighted in red color.

\section{DECLARATIONS}

\section{Authors' contributions}

Conceptualization, methodology, validation, visualization: Verma S, Gupta S

Writing original draft, review, and editing: Verma S, Gupta S

Software, formal analysis, investigation, data curation: Verma S

Resources, supervision, project administration, funding acquisition: Gupta $S$

Both authors have read and agreed to the published version of the manuscript.

\section{Availability of data and materials}

GSE150368 and GSE69223 and open data sources used to build the hypothesis. These data sources are available on the website at https://www.ncbi.nlm.nih.gov/gds/.

\section{Financial support and sponsorship}

None.

\section{Conflicts of interest}

Both authors declare that there are no conflicts of interest.

\section{Ethical approval and consent to participate}

Not applicable. 


\section{Consent for publication}

Not applicable.

\section{Copyright}

(c) The Author(s) 2021.

\section{REFERENCES}

1. Shang J, Wan Y, Luo C, et al. Cell entry mechanisms of SARS-CoV-2. Proc Natl Acad Sci U S A 2020;117:11727-34. DOI PubMed PMC

2. Tomlins SA, Rhodes DR, Perner S, et al. Recurrent fusion of TMPRSS2 and ETS transcription factor genes in prostate cancer. Science 2005;310:644-8. DOI PubMed

3. Clinckemalie L, Spans L, Dubois V, et al. Androgen regulation of the TMPRSS2 gene and the effect of a SNP in an androgen response element. Mol Endocrinol 2013;27:2028-40. DOI PubMed PMC

4. Patel VG, Zhong X, Liaw B, et al. Does androgen deprivation therapy protect against severe complications from COVID-19? Ann Oncol 2020;31:1419-20. DOI PubMed PMC

5. Montopoli M, Zumerle S, Vettor R, et al. Androgen-deprivation therapies for prostate cancer and risk of infection by SARS-CoV-2: a population-based study ( $\mathrm{N}=4532)$. Ann Oncol 2020;31:1040-5. DOI PubMed PMC

6. Kwon DH, Vashisht R, Borno HT, et al. Androgen-deprivation therapy and SARS-CoV-2 in men with prostate cancer: findings from the University of California Health System registry. Ann Oncol 2021;32:678-9. DOI PubMed PMC

7. Klein EA, Li J, Milinovich A, et al. Androgen deprivation therapy in men with prostate cancer does not affect risk of infection with SARS-CoV-2. J Urol 2021;205:441-3. DOI PubMed

8. Long X, Hou H, Wang X, et al. Immune signature driven by ADT-induced immune microenvironment remodeling in prostate cancer is correlated with recurrence-free survival and immune infiltration. Cell Death Dis 2020;11:779. DOI PubMed PMC

9. Meller S, Meyer HA, Bethan B, et al. Integration of tissue metabolomics, transcriptomics and immunohistochemistry reveals ERGand gleason score-specific metabolomic alterations in prostate cancer. Oncotarget 2016;7:1421-38. DOI PubMed PMC

10. Qiao Y, Wang XM, Mannan R, et al. Targeting transcriptional regulation of SARS-CoV-2 entry factors ACE2 and TMPRSS2. Proc Natl Acad Sci U S A 2020;118:e2021450118. DOI PubMed PMC

11. Ziegler CGK, Allon SJ, Nyquist SK, et al. SARS-CoV-2 receptor ACE2 is an interferon-stimulated gene in human airway epithelial cells and is detected in specific cell subsets across tissues. Cell 2020;181:1016-35.e19. DOI PubMed PMC

12. Catanzaro JM, Sheshadri N, Pan JA, et al. Oncogenic Ras induces inflammatory cytokine production by upregulating the squamous cell carcinoma antigens SerpinB3/B4. Nat Commun 2014;5:3729. DOI PubMed PMC

13. Sheshadri N, Catanzaro JM, Bott AJ, et al. SCCA1/SERPINB3 promotes oncogenesis and epithelial-mesenchymal transition via the unfolded protein response and IL6 signaling. Cancer Res 2014;74:6318-29. DOI PubMed PMC 